\title{
ESTIMATION OF SIGNAL PARAMETERS FOR MULTIPLE TARGET LOCALIZATION
}

\author{
X. Susan Christina ${ }^{1}$, S. Ramya $^{2}$ and S. Maheswari ${ }^{3}$ \\ ${ }^{I}$ Department of Electronics and Communication Engineering, MIET Engineering College, India \\ E-mail: fab_jesu@yahoo.co.in \\ ${ }^{2,3}$ Department of Electronics and Communication Engineering, Mookambigai College of Engineering, India \\ E-mail: ${ }^{2}$ sramya0290@gmail.com, ${ }^{3}$ meenamahasri@gmail.com
}

\begin{abstract}
Target detection and localization is an active research area due to its importance in a wide range of application such as biomedical and military applications. In this paper, a novel method for the detection and estimation of signal parameters such as range and direction of arrival for multiple far-field target using wideband echo chirp signals is proposed. Sonar and radar are the active detection systems transmit well defined signals into a region of interest. A model preprocessing procedure is designed for the echo signal. The parameters estimation method for multiple targets is developed based on the Linear Canonical transform and the Fast Root MUSIC algorithm which is a high resolution DOA estimation method originally proposed for any arbitrary arrays to reduce the computational complexity in existing systems. The proposed method provides high accuracy of detection and resolution even in very low SNR values.
\end{abstract}

Keywords:

Active Detection, Location Estimation, Linear Canonical Transform

\section{INTRODUCTION}

Accurate localization of multiple targets is one of the fundamental and challenging problems in signal processing. Due to its importance in a wide range of applications such as military and biomedical applications, it provides an active research area. In this paper, the problem of localizing multiple far-field targets using radar system is addressed. In active radar system, a known waveform is transmitted and the signal reflected from the target of interest is used to estimate its parameters. Typically, the received signal is modeled as a scaled, delayed, and Doppler-shifted version of the transmitted signal. Estimation of the time delay and Doppler shift provides information about the range and radial velocity of the target [1]. Linearly frequency modulated (LFM) signals are often used in active detection systems as they provide good target detection sensitivity of low Doppler targets while having high range resolution [2].

Target range and velocity estimation of moving targets using high resolution is a topic of great interest. To achieve this goal, there are two problems to be considered. The first problem is how to obtain high range resolution. It is known that the range resolution is directly proportional to bandwidth of the transmitting signal. So ultrawideband signal processing is a well known technique to obtain high range resolution. The second problem is how correctly the range and velocity of the moving target measure [3]. When the target of interest is stationary, its position can be estimated by inverse discrete Fourier transform on the received echo signal. However, when the target is moving, it is necessary to deal with following difficulties. Firstly, the radial velocity of the target may cause range estimate shifted, which is called range-Doppler coupling, and both range position and radial velocity cannot be correctly retrieved in the received signal. Secondly, for high speed target such as airplane or missile, the received signal in the form of $2 \pi$ modulo folding called phase wrapping [4]. Fast root-MUSIC algorithm is used to robustly resolve both the range-Doppler coupling and the phase wrapping. The radar transmits and receives wideband signal with different carrier frequencies and the linear canonical transform is operated on every pulse train [5].

Generally the estimation of the direction of arrival (DOA) of a target is the classical delay and sum beamforming, but it results in low resolution and high side lobe levels. Multiple signal classification (MUSIC) and estimation of signal parameters via rotational invariance techniques (ESPRIT) provides high resolution DOA estimation of multiple targets, but they were only applicable to narrowband applications. For wideband signals, DOA estimators based on fast root-MUSIC method is used.

The rotation of the time-frequency plane could be carried out to improve the energy concentration of signals which are not sparse in either the time or the frequency domain. For analyzing chirp signals, Radon ambiguity and Radon-Wigner transforms were useful but they suffer from cross-terms and hence high computational complexity. The Linear canonical transform which is gaining much attention recently is a signal processing tool based on the rotation of the timefrequency plane [6]. It is a generalization of the Fractional Fourier transform and is a representation of signals using an orthonormal basis formed by chirps. It is therefore expected that the multiple chirp signals can be conveniently separated in a carefully chosen domain.

In this paper, we propose an efficient method for estimating the location and radial velocities of multiple targets from the received echo chirp signals and the block diagram is shown in Fig.1.

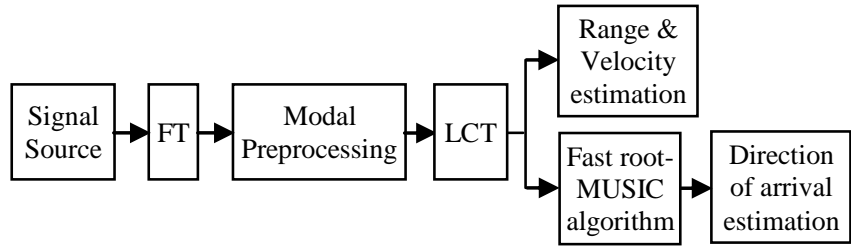

Fig.1. The block diagram of the proposed method for simultaneously estimating the range, velocity and direction of arrival of multiple targets

A wideband signal is used to transmit the signal in a far field area and then a modal preprocessing step is carried out to transform the received signals at different modes. By further processing the signals at different modes, the parameters of the targets are estimated. The properties of the linear canonical transform of chirps are used to estimate the range and radial velocity of the targets while the DOA of each of the targets can be estimated using fast root-MUSIC algorithm. 
The paper is organized as follows: Radar model of an active detection system is described in section 2. The definition of the linear canonical transform and the properties are discussed in section 3. Section 4 describes how the parameters of the targets can be estimated from the signals at the different modes. Section 5 describes the result obtained in simulation. The final section summarizes the main contribution of this paper.

\section{RADAR MODEL}

Radar which is an active detection system transmits a well defined signal into a region of interest and by processing the echoes reflected from the targets present, detects and estimates the signal parameters such as range, velocity and direction of arrival [7]. The total number of target in the region of interest be denoted by $P$. At a particular time instance, each of the $P$ targets is located at a range $D_{p}$ at direction $u_{p}$ from the transmitter and each of them moves with an independent constant velocity $V_{T p}$.

Let the transmitted signal be $s(t)$. Following the slowly fluctuating transmitted signal and assuming that the targets are located in the far field, the received signal at the antenna at time can be written as,

$$
x_{q}(t)=\sum_{p=1}^{P} \gamma_{p} S_{p}\left(t+\tau_{p q}\right)+n_{q}(t)
$$

where, $\gamma_{p}$ is the range dependent attenuation factor in the propagation path to and back from the $p^{\text {th }}$ target, $\tau_{p q}=h_{\mathrm{q}} \cdot u_{\mathrm{p}} / c$ is the propagation delay of the received signal from the $p^{\text {th }}$ target with $c$ being the speed of signal propagation.

\section{LINEAR CANONICAL TRANSFROM(LCT)}

The linear canonical transform (LCT) is a much more general integral transform which has the Fourier transform, Fractional Fourier transform, Fresnel transform etc as its special cases. As it is well known that fractional Fourier transform is the powerful mathematical tool and is widely used for spectral analysis, signal processing, optical system analysis etc., the LCT which is the generalization of FrFT has obviously more ability and potential due to its four parameters ( $a, b, c, d)$ which has the ability to change the values based on the need.

The LCT of a signal $x(t)$ is defined as,

$$
L^{(a, b, c, d)}[f(t)](u)=\left\{\begin{array}{l}
\sqrt{\frac{1}{j 2 \pi b}} e^{j \frac{d}{2 b}} u^{2} \int_{-\infty}^{\infty} \exp \left(j \frac{a}{2 b} t^{2}-j \frac{u t}{b} f(t) d t(b=0)\right) \\
\sqrt{d e} j \frac{c d}{2} u^{2} f(d u)(b \neq 0)
\end{array}\right.
$$

where, $a, b, c, d$ are the real numbers and satisfying $a d-b c=1$. It is easy to verify that the classical FT, FrFT, the chirp operation and the scaling operation are all the special cases of the LCT as shown in the following:

1) When $(a, b, c, d)=(0,1,-1,0)$, the LCT becomes the FT:

$$
L^{(0,1,-1,0)}[f(t)](u)=\sqrt{-j} F T[f(t))(u)
$$

2) When $(a, b, c, d)=(\cos \theta, \sin \theta,-\sin \theta,-\cos \theta)$, the LCT becomes FrFT:

$$
L^{(\cos \theta, \sin \theta,-\sin \theta,-\cos \theta)}[f(t)](u)=\sqrt{e^{-j \alpha}} F^{\alpha}[f(t)]
$$

3) When $(a, b, c, d)=(1,0, \tau, 1)$, the LCT becomes the chirp operation:

$$
L^{(1,0, \tau, 1)}[f(t)](u)=e^{\frac{j \tau u^{2}}{2}} f(u)
$$

4) When $(a, b, c, d)=(\sigma, 0,0, \sigma-1)$, the LCT becomes the scaling operation:

$$
L^{(\sigma, 0,0, \sigma-1)}[f(t)](u)=\sqrt{(\sigma-1)} f\left(\sigma^{-1} u\right)
$$

It shows that the LCT is one of the most important non stationary signal processing tools applicable in many areas. It can be seen that the magnitude of the LCT of the delayed signal is a delayed version of the magnitude LCT of the original signal, and the delay observed in the LCT is linearly proportional to $\tau$. Therefore, the LCT would be powerful signal processing tool to estimate the time of arrival of signals at the receiving end.

It not only handles a much more general family of integrals, but also effectively addresses certain difficulties, limitation and trade off during the separation of overlapped signal. Hence, applications benefiting from the LCT require minimal additional implementation cost compared to FrFT.

Separation of the individual LFM signals [8] from the composite signal is a three stage process:

1) LCT is performed on the composite signal transforming the overlapping LFM signals in the time domain into separable pulses in the fractional domain.

2) The individual pulses are identified and windowed creating multiple signals each containing one pulse.

3) The signals containing the windowed pulses are restored to the time domain using LCT and thus the individual LFM signals are separated.

The Fig.2 illustrates the signal flow diagram for separation of temporally overlapping LFM signal using the LCT. The optimum transform order $\alpha_{o p t}$ can be defined as

$$
\alpha_{\text {opt }}=-(2 / \pi) \tan ^{-1}(1 / 2 a)
$$

where, $a=B / T$ is the chirp rate, $B$ is the bandwidth in Hertz and $T$ is the total signal duration in seconds. It should be noted that calculation of $\alpha_{\text {opt }}$ only requires knowledge of the chirp rate, a known excitaion parameter.

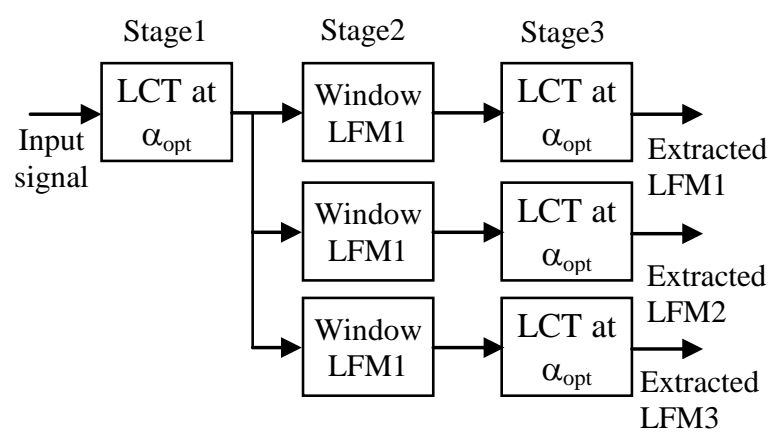

Fig.2. Signal flow diagram for seperation of temprorally overlapping LFM signals using theLCT 


\section{OBSERVABLE TARGET PARAMETERS}

In this section, we describe how the parameters (range, radial velocity and DOA) of the targets present can be estimated from the received signals. The radar is used to detect objects in the observation area and to retrieve the objects. The waveform plays an important role in the ability of the radar to detect objects and measure their parameters. For detection and measurements the radar transmits the waveform and interprets the objects backscattered echo signals. The signals at different modes can be written as,

$$
Z(\omega)=A S(\omega)+N(\omega)
$$

where, $N(\omega)$ is the noise at the different modes. These signals at the different modes are in a form that can be further processed for multiple target detection and localization. The proposed parameter estimation method is developed based on the properties of the linear canonical transform of chirps and fast root-MUSIC, which provides high resolution DOA estimation of signals received at arbitrary array.

\subsection{TARGET RANGE}

The target range parameter $\mathrm{R}$ refers to the distance between the radar antenna and the target [9]. The target range is proportional to the time $\tau$ it takes the signal to travel from the radar transmitter to the reflecting target and back to the radar receiver:

$$
R=(c \cdot \tau) / 2
$$

where, $c$ is the speed of light.

\subsection{TARGET VELOCITY}

The relative radial velocity $v_{r}$ between the target and radar is determined by the frequency of a received sound wave is changed depending on the relative velocity between transmitter and receiver called Doppler Effect [10]. It is caused by the continuous change of distance between the radar and the target that squeezes or expands the received signal in time. Assuming a target at initial range $R_{0}$ with constant radial velocity $v_{r}$, the received signal $r(t)$ is delayed by the time dependent signal propagation delay $\tau(t) \approx$ $\left[2\left(R_{0}+v_{r} t\right)\right] / c$. in the case of a transmit signal $s(t)$ being a harmonic oscillation with frequency $f_{c}$, the Doppler shift of the received oscillation can be determined as,

$$
\begin{gathered}
s(t)=\sin \left(2 \pi f_{c} t\right) \\
r(t) \approx s\left(t-\frac{2\left(R_{0}+v_{r} t\right)}{c}\right) \\
r(t) \approx \sin \left(2 \pi f_{c}\left(t-\frac{2\left(R_{0}+v_{r} t\right)}{c}\right)\right)
\end{gathered}
$$

The middle term of the sine function argument contains the Doppler frequency shift:

$$
f_{D} \approx-\frac{2 f_{c} v_{r}}{c}=-\frac{2 v_{r}}{\lambda}
$$

where, $\lambda$ is the signal's wavelength. Only the radial component of the relative target velocity has an impact on the Doppler frequency.

A linear chirp pulse is transmitted isotropically and the echoes reflected from the independent and distinct targets in vicinity are processed to estimate their positions and velocities. The reflected signal from the target is defined as,

$$
S_{p}(t)=S\left(\sigma_{p} t-\tau_{p}\right)
$$

where, $\tau_{p}=2 D_{p} / c$ is the delay parameter of the $p^{\text {th }}$ target and $\sigma_{p}$ $=1+2 v_{p} / c$ is the time compression/stretching parameter of the $p^{\text {th }}$ target. It is a scaled and delayed version of the original transmitted signal, associated with the $p^{\text {th }}$ target as seen at the origin of receiver end.

The received signal at each mode is a summation of the signals associated with each target multiplied by a complex exponential term dependent on the mode number and DOA of the target. The magnitude of the LCT of a received echo chirp signal has a mainlobe with a width and center dependent on the delay parameter $\tau$ and the compression/stretching parameter $\sigma$ associated with the range and velocity of the target reflecting the signal [11]. Therefore, by estimating the locations and widths of the peaks detected in the magnitude of the received signal, the range and radial velocity of each target from the origin of the receiving end can be estimated.

\subsection{DIRECTION OF ARRIVAL (DOA) ESTIMATION}

Direction of arrival estimation is an important algorithm in array processing. Most traditional DOA estimation methods focus on narrow band sources. Wideband DOA estimation, echo gain at each direction is calculated based on group delay compensation. Estimation methods such as maximum likelihood (ML), multiple signal classification (MUSIC) [12] and ESPRIT [13] are based on narrowband model which provides low resolution. In this section, a DOA estimation method based on fast root-MUSIC for wideband signal which provides high resolution estimation using linear canonical transform which has the capability of separating chirp signals for arbitrary arrays.

Consider an array of $N$ antenna element operating in the presence of $M$ uncorrelated wideband sources. By using manifold separation technique (MST) [14], the manifold vector $S(r, \theta)$ can be written as,

$$
\underline{S}(r, \theta)=G(r) \underline{d}(\theta)+\underline{\varepsilon}
$$

where, the matrix $G(r)$ depends on array geometry only. The Vandermonde structured vector $\underline{d}(\theta)$ is a function of direction of arrival (DOA) only, defined as,

$$
\underline{d}(\theta)=\frac{e^{j(q-1 / 2) \theta}}{\sqrt{2 \pi}}\left[1, z, . . z^{(q-1)}\right]^{T}
$$

where, $z=e^{-j \theta}$ and $\theta$ is the DOA. The modeling error $\underline{\varepsilon}$ can be safely neglected, provided that $q$ is a sufficiently large number. A polynomial is constructed as follows:

$$
f(z)=\frac{1}{2 \pi} \sum_{i=-(q-1)}^{q-1} b_{i} z^{-i}
$$

Taking into account the Hermitian property, one obtains $b_{i}=b_{-i}^{*}$ where, (.)* represents complex conjugate operation. This implies that $f(z)$ is a Laurent polynomial. Also $f(z)$ is non-negative because $f(z)=\left\|E_{n}^{H} \underline{S}(r, \theta)\right\|^{2} \geq 0$, where, $\|$.$\| denotes the Euclidean$ norm of a vector, then $f(z)$ can be factorized as, 


$$
f(z)=c_{1} f_{1}(z) f_{1}^{*}\left(1 / z^{*}\right)
$$

where, $c_{1}$ is a positive constant. The roots of $f(z)$ appear in conjugate reciprocal pairs, i.e. if $z_{1}$ is a root of $f(z)$, then $\left(z_{1}^{-1}\right)^{*}$ is also a root. This property suggests that computing half of the roots (i.e. roots of $f_{1}(z)$ ) is sufficient to find the roots of interest. A fast spectral factorization method based on the Schur algorithm is applied, which can be implemented in the following steps:

1) Initialize a $(Q \times 2)$ matrix $B_{0}$ as,

$$
B_{k}=\left(\begin{array}{cc}
b_{0} b_{-1} \ldots & b_{-}(Q-1) \\
b_{-1} b_{-2} \ldots & b_{0}
\end{array}\right)^{T} \text { for } k=0
$$

2) For $k=1,2$. Until convergence, iterate the following steps: a) $B_{k}=B_{k-1} U_{k}$, where $U_{k}$ is a $(2 \times 2)$ matrix defined as,

$$
U_{k}=\frac{1}{\sqrt{1-|\gamma|}^{2}}\left(\begin{array}{cc}
1 & -\gamma \\
\gamma & 1
\end{array}\right)
$$

with $\lambda=\left[B_{k-1}\right]_{1,2} /\left[B_{k-1}\right]_{1,1}$, i.e. the ratio of the two entries of the first row of $B_{k-1}$.

b) Shift up the second column of $B_{k}$ by one element while keeping the first column unaltered

c) Test for convergence $\left\|\underline{b}_{1, k}-\underline{b}_{1, k-1}\right\|<$ threshold, where, $\underline{b}_{1, k}$ and $\underline{b}_{1, k-1}$ denote the first column of $B_{k}$ and $B_{k-1}$, respectively. If converged, go to step(3), else return to step $2 \mathrm{a}$.

3) The coefficient of $f_{1}(z)$ are $b_{1, k}^{*}$.

Now the polynomial factor $f_{1}(z)$ which has all its roots on or inside the unit circle, is obtained. To find the roots one can construct an unsymmetric companion matrix $M$ the Eigenvalues of which correspond to the roots of $f_{1}(z)$. Because the Eigenvalues of interest must be the largest ones, one can make use of the Arnoldi iteration to calculate only the $M$ largest Eigenvalues.

The fast root-MUSIC algorithm for arbitrary arrays can be accomplished via the following steps:

1) Compute the sampling matrix $G(r)$.

2) Form the received data covariance matrix and perform Eigen value decomposition.

3) Perform fast spectral factorization on $f(z)$ via the Schur algorithm to obtain the polynomial factor $f_{1}(z)$ and the companion matrix $M$.

4) Apply the Arnoldi iteration method to calculate the $M$ largest Eigenvalues of $M$, then DOAs can be estimated by the phase angles of these Eigenvalues.

Rather than computing all the roots as in the conventional approaches, the proposed fast root-MUSIC algorithm computes only the roots of interest (those corresponding to the true DOAs).

\section{RESULTS AND DISCUSSION}

In order to verify the results in this article and show the advantage of the LCT in the signal detection, the simulations are performed in this section. From these simulations, it is easy to see that the LCT has a better performance in detections of the target than the traditional one. The performance metric used is the root mean square error for each parameter estimated for each of the $P$ targets, which is defined as,

$$
R M S_{p}=\sqrt{\frac{1}{M}} \sum_{m=1}^{M}\left\|\hat{\mu}_{p}^{m}-\mu_{p}\right\|^{2}
$$

where, $\mu_{p}$ and $\hat{\mu}_{p}$ are the true and estimated parameter of the $P^{\text {th }}$ target in the $m^{\text {th }}$ run and $M$ is the total number of Monte Carlo runs. The parameters $\mu$ tested are the range $D$, radial velocity $v$ and DOA $\hat{\mu}_{p}$. Therefore, in our simulations here, the performance of the proposed estimation is compared with the fractional Fourier transform based method for single or multiple sources.

\subsection{SINGLE TARGET}

The capability of estimating the root mean square error (RMS) for single target is shown in Figs.3, 4 and 5. It can be seen that using the proposed method, all the parameters of the target can be estimated with acceptable accuracy even at low SNR values.

In Fig.3, estimation of range for single target is compared with existing algorithm. The RMS error of single target is maintained constant at around $3 \mathrm{~dB}$.

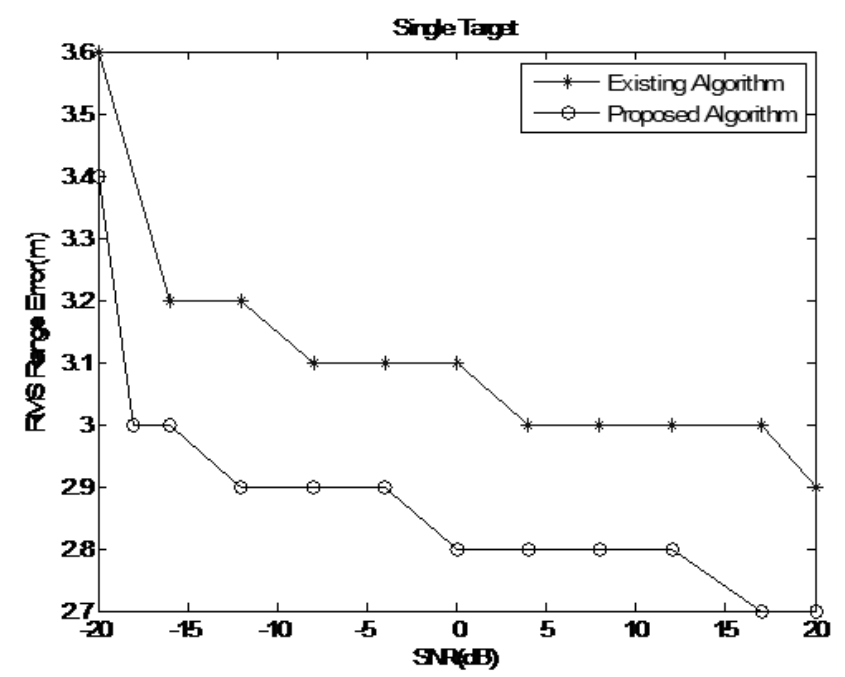

Fig.3. The RMS range error for single targets over different SNR values

The Fig.4 shows the estimation of velocity for single target having different SNR values. The proposed method reduces the RMS error by $0.04 \mathrm{~dB}$ and maintained constant at $13 \mathrm{~dB}$ SNR value.

Besides that, the proposed algorithm is computationally less complex than the existing algorithm as it does not involve a search over a range of transform angles to estimate the range and velocity of the target.

The root- MUSIC based DOA estimation method has similar performance to the proposed method in high SNR cases $(>15 \mathrm{~dB})$. However, its error increases more rapidly compared to the proposed method when the SNR value decreases. 


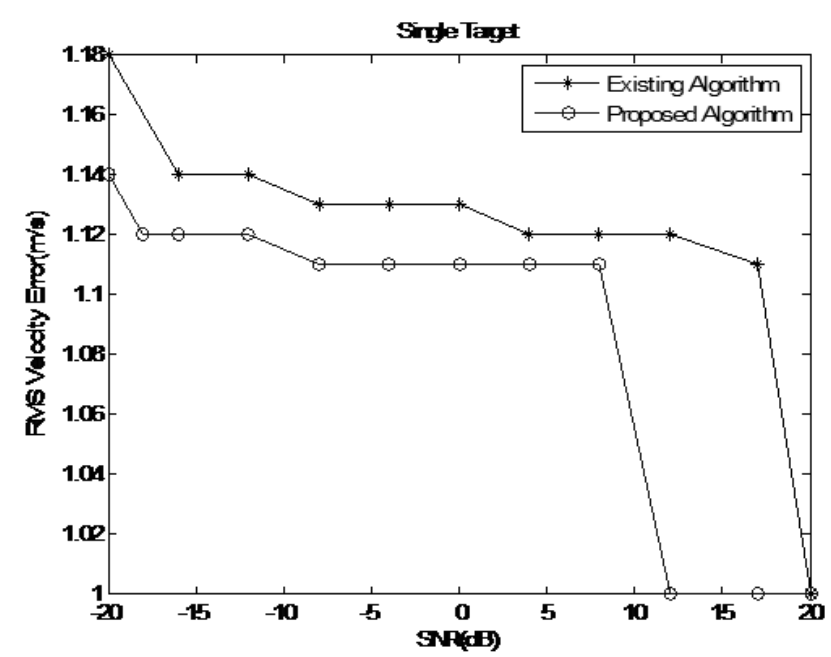

Fig.4. The RMS velocity error for single targets over different SNR values

The Fig.5 demonstrate the estimation of direction of arrival for single target.

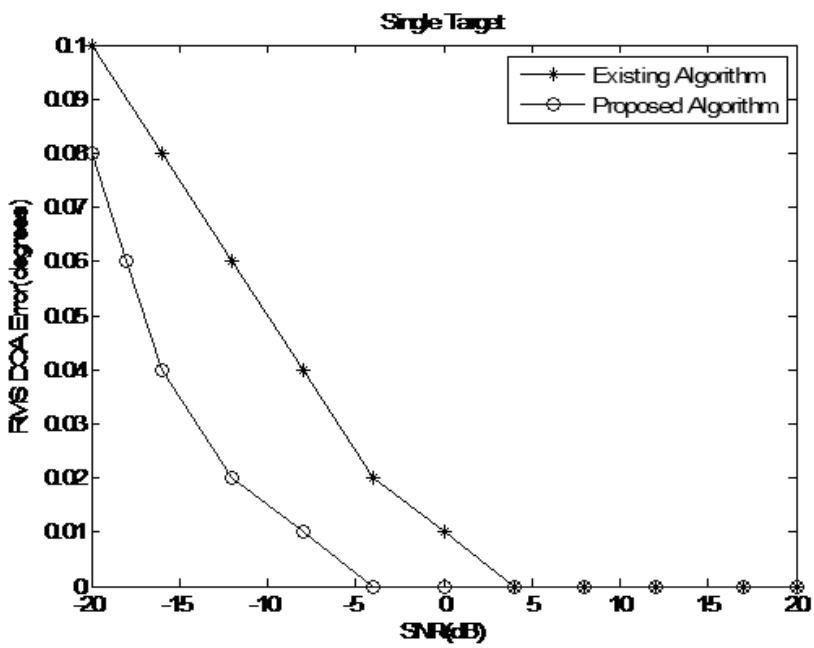

Fig.5. The DOA error of two closely spaced targets over different SNR values

\subsection{TWO CLOSELY SPACED TARGET}

The capabilities of the proposed method in separating two closely spaced targets are then examined. In the Cartesian coordinates, the target is originally located at $[100,100] \mathrm{km}$ and the RMS error for range of the proposed method is shown in Fig.6.

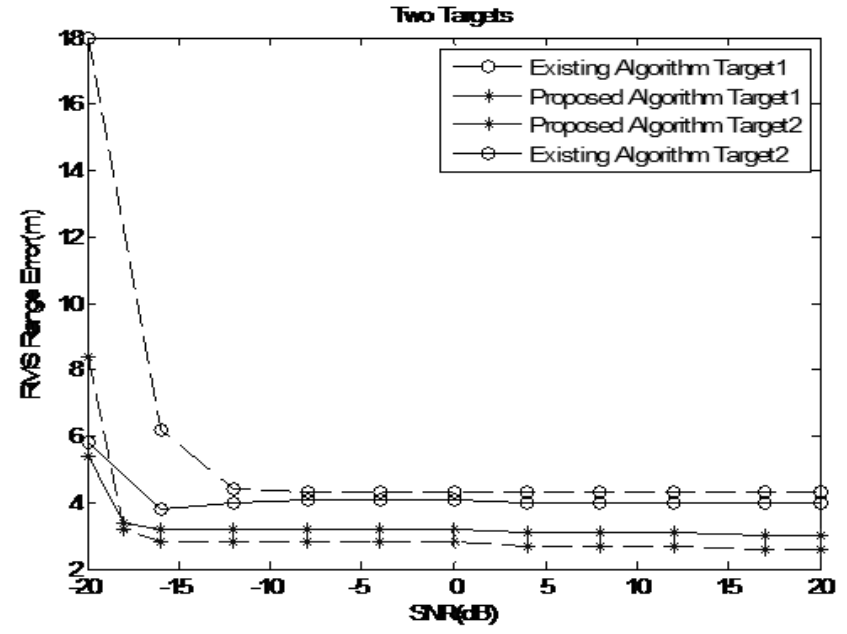

Fig.6. The RMS range error of two targets over different SNR values

Here improvement in RMS error in a range of about $2 \mathrm{~dB}$. However it shows the capability for estimating even in low SNR values below $-10 \mathrm{~dB}$.

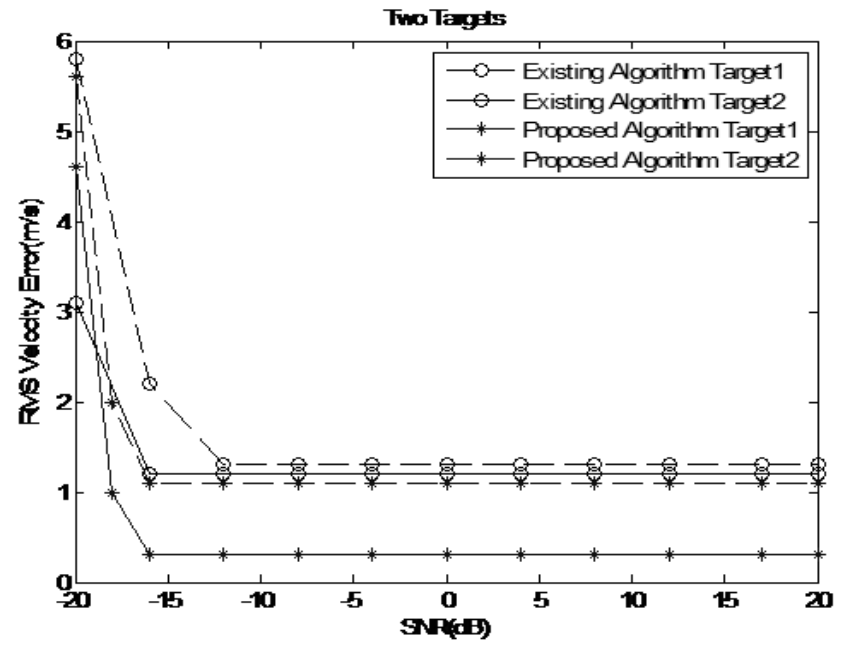

Fig.7. The RMS range error of two closely spaced targets over different SNR values

In Fig.7, the velocity error of closely spaced target is estimated. It can be shown that using the proposed method, all the parameters of the target can be estimated with acceptable accuracy even at low SNR values. The DOA of each of the two targets over a range of SNR values are shown in Fig.8. 


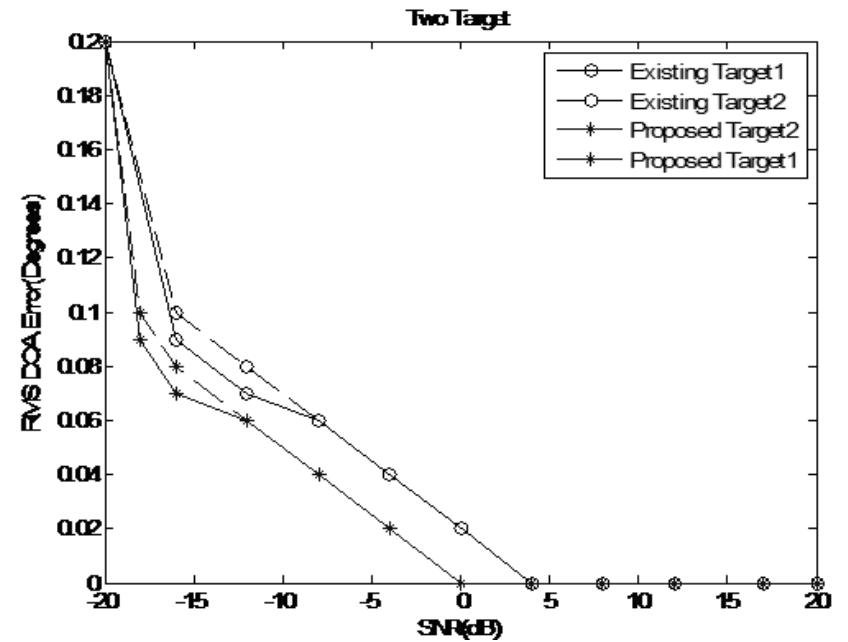

Fig.8. The RMS DOA error of two closely spaced targets over different SNR values

It can be produce the result even at low SNR values below $10 \mathrm{~dB}$. By using the proposed method, the RMS errors of the estimated range and DOA are clearly determined.

\subsection{P>2 TARGET}

The RMS performance curves of the proposed method in estimating the parameters of a number of $P>2$ targets is similar to that shown for a single and two closely spaced targets. The error for each of the target is shown in Fig.9.

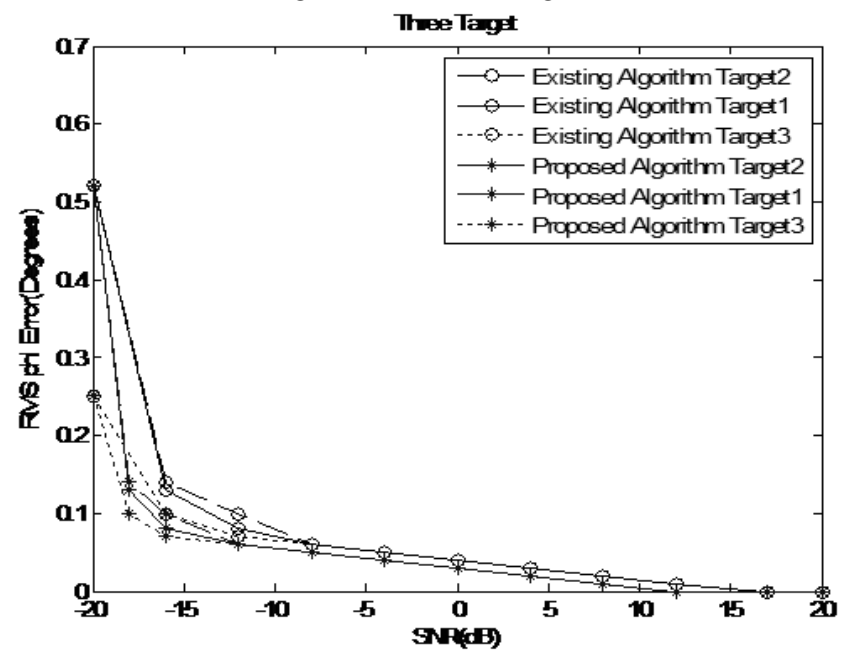

Fig.9. The RMS DOA error of three targets over different SNR values

There is no limit on the number of targets detectable as long as the signals associated with each independent target is separable in the Linear canonical transform domain of the transmitted signal.

\section{CONCLUSION}

In this paper, the multiple target localization based on linear canonical transform is presented. It is especially applicable in fast, time-varying environments, where multiple targets maneuver quickly and randomly. A modal preprocessing technique allows fast direction of arrival estimation designed for arbitrary configurations. It can be shown that the proposed method outperforms both the conventional FrFT and root-MUSIC technique. Simulation result showing the accuracy of the proposed analysis is presented.

\section{REFERENCES}

[1] Ioannis Bertsatos and Nicholas C. Makris, "Estimating the instantaneous velocity of randomly moving target swarms in a stratified ocean waveguide by Doppler analysis", The Journal of the Acoustical Society of America, Vol. 130, No. 1, pp. 84-101, 2011.

[2] D. M. J. Cowell and S. Freear, "Separation of Overlapping Linear Frequency Modulated (LFM) signals using the Fractional Fourier Domain", IEEE Transactions on Ultrasonics, Ferroelectrics and Frequency Control, Vol. 57, No. 10, pp. 2324-2333, 2010.

[3] J. Jayanti Sharma, Christoph H. Gierull and Micheal J. Collins, "The influence of target acceleration on velocity estimation in dual channel SAR-GMTI", IEEE Transaction on Geosciences and Remote Sensing, Vol. 44, No.1, pp. 134147, 2006.

[4] Gang Li, Huadong Meng, Xiang Gen Xia and Ying Ning Peng, "Range and velocity estimation of moving targets using multiple stepped frequency pulse trains", Sensors, Vol. 8, No. 2, pp. 1343-1350. 2008,

[5] J. Zhuang, W. Li and A. Manikas, "Fast root-MUSIC for arbitrary arrays", Electronic Letters, Vol. 46, No. 2, pp.174$176,2010$.

[6] Healy John J and Sheridan John T, "Fast Linear Canonical Transform", Optical Society of America, Vol. 27, No 1, pp. 13-18, 2010.

[7] Xin Yuan, "Direction-Finding wideband Linear FM Sources with triangular arrays", IEEE Transactions on Aerospace and Electronic Systems, Vol. 48, No. 3, pp. 2416-2425, 2012.

[8] Volkan Cevher, Velmurugan Rajbabu and McClellan H. James, "A range-only multiple target particle filter tracker", Proceedings of the IEEE International Conference on Acoustics, Speech and Signal Processing, Vol. 4, pp. IV, 2006.

[9] Jens E. Wilhjelm and Peder C Pedersen, "Target velocity estimation with FM and PW Echo ranging Doppler systemspart-2: system analysis", IEEE Transactions on Ultrasonics, Ferroelectrics and Frequency Control, Vol. 40, No. 4, pp. 373-380, 1993.

[10] Xinrong $\mathrm{Li}$, "An Iterative NLOS mitigation algorithm for location estimation in sensor networks", Proceedings of the $15^{\text {th }}$ IST Mobile and Wireless Communication Summit, 2006.

[11] Ralph O. Schmidt, "Multiple Emitter Location and Signal Parameter Estimations", IEEE Transaction on Antennas and Propagation, Vol. 34, No. 3, pp. 276-280, 1986.

[12] Richard Roy and Thomas Kailath, "ESPRIT-Estimation of signal parameters via rotational invariance techniques", IEEE Transaction on Acoustics, Speech and Signal Processing, Vol. 37, No. 7, pp. 984-995, 1989.

[13] Fabio Belloni, Andreas Richter and Visa Koivunen, "DOA Estimation via Manifold Separations for arbitrary array structures", IEEE Transactions on Signal Processing, Vol. 55, No. 10, pp. 4800-4810, 2007. 